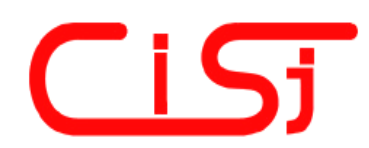

\title{
WIRELESS DATA ACQUISITION SYSTEM USING BLUETOOTH TECHNOLOGY FOR INFRASONIC RECORDS
}

\author{
José Chilo ${ }^{1)}$, Thomas Lindblad ${ }^{2)}$ \\ 1) University of Gävle, S-80176, Sweden (e-mail: jco@hig.se) \\ ${ }^{2)}$ Royal Institute of Technology, S-10691, Sweden (e-mail: lindblad@particle.kth.se)
}

\begin{abstract}
Advancements in electronics provide a vital new option for implementation of low-frequency smart sensors that can perform signal processing close to the sensors and transmit the data wirelessly. These smart sensors can improve the efficiency of an automatic classification system and reduce the cost of actual infrasound microphones. The design of a digital wireless data acquisition system using a QF4512 programmable signal converter from Quickfilter Technologies, a MSP430 microcontroller from Texas Instruments and a F2M03GLA Bluetooth module from Free2move for infrasonic records is presented in this paper. The digital wireless data acquisition system has passed extensive laboratory and field tests (e.g. with man-made explosions).
\end{abstract}

Keywords: Bluetooth, infrasound, acquisition system, digital filtering.

\section{INTRODUCTION}

Infrasound is low frequency sound, typically of a frequency of a few Hertz to 20 Hertz. Due to its inherent properties, infrasound can travel distances of many hundreds of kilometers. Infrasound signals can result from nuclear explosions, volcanic eruptions, mountain associated waves, auroral waves, earthquakes, meteors, avalanches, severe weather, quarry blasting, air/spacecraft, gravity waves, microbaroms, opening and closing of doors, trains and helicopters to name a few. The infrasonic frequency band recently gained renewed attention because of the Comprehensive Nuclear-Test-Ban Treaty (CTBT) [1] and others, in particular infrasound networks connected to Internet [2].

However, infrasound propagating long distances is a complex phenomenon. It is strongly influenced by the detailed temperature and wind profiles. The infrasonic signal detected by traditional infrasound systems contains the combination of the source's infrasound power spectrum and the distortions introduced by the atmosphere. In order to extract the source characteristics from the collected data, advanced signal processing is required. In this paper we present a Wireless Digital Data Acquisition System that can be used for detecting, locating, and identifying infrasound sources at close range: from a few meters to a few km distances. At short distances, the atmosphere is a homogeneous medium that preserves the infrasonic waveform as it is generated by the source. In this system the signal processing is performed close to the sensors.

\section{DATA ACQUISITION}

We would like to design a system that is portable, easy to install, low cost, low power consuming and that it can be used in hostile environments. In order to address these requirements a Wireless Digital Data Acquisition System as shown in Fig. 1 is proposed. The design of this system features data taking from four element microphones, analog signal conditioning, analog to digital conversion, digital filtering and storage of the data before it is sent out using wireless means. This design approach is based on the QF4A512 Programmable Signal Converter developed by Quickfilter Technologies [3-4], the MSP430 microcontroller from Texas Instruments and a F2M03GLA Bluetooth module from Free2move [5]. The QF4A512 handles four sensor signals simultaneously.
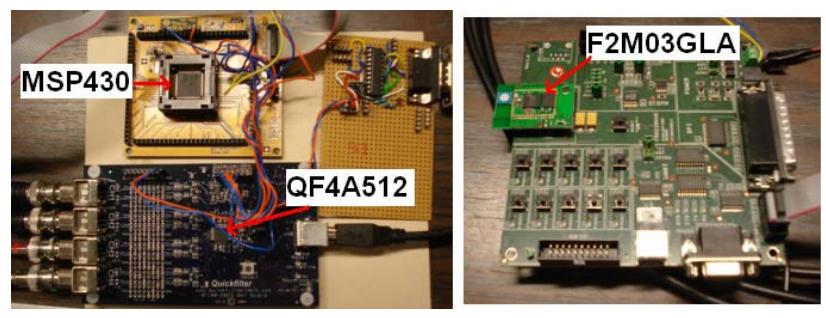

Fig. 1 - The proposed Data Acquisition System. 
The infrasonic sensors used in our experiments are four MCE-200 element microphones from Panasonic. These element microphones have a flat response function in the audible band (from $>20 \mathrm{~Hz}$ to $16 \mathrm{kHz}$ ) and operation in the infrasound band (below $20 \mathrm{~Hz}$ ) is outside the manufacturer's specified range of operation. However, these element microphones have been used in many infrasound networks, see Fig. 2. The four redundant element microphones help to achieve heightened signal-to-noise ratio and provide robustness in case one of them should fail. We should note that these element microphones could be replaced with any better infrasonic sensors.

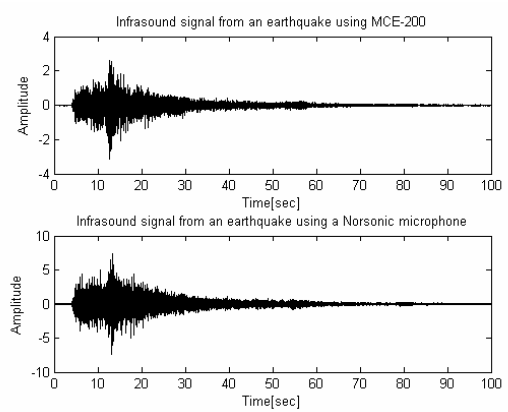

Fig. 2 - A MCE-200 microphone and a Norsonic microphone.

\subsection{THE QF4A512 PROGRAMMABLE SIGNAL CONVERTER}

The functional circuit blocks for processing the signals from the element microphones using the QF4A512 are shown in Fig. 3. The first link is a signal conditioning analog front-end Programmable Gain Amplifiers (PGA) and Anti-Aliasing Filter (AAF). The second link is the all important Analog to Digital Converter (ADC) itself. The ADC can be programmable to cover a wide range of frequency inputs. The third link represents a piece of signal processing in the form of digital Finite Impulse Response (FIR) filter.

The signals output from the microphones are analog and can vary in voltage and frequency range depending on the type of event. These signals are then amplified and the amount of gain chosen can be $1 \mathrm{x}, 2 \mathrm{x}, \ldots, 8 \mathrm{x}$. Next the signals are converted to digital via a 16-bits ADC. To prevent errors in the conversion process, low-pass Anti-Aliasing Filters are employed prior to the ADC. The operating frequency of the ADC is also selected specifically for the signal frequency coming from the sensor. Digital filtering is performed after the ADC using a Digital Signal Processor (DSP). This filtering is more accurate than the filtering of the analog signal before the ADC. Each signal path has a dedicated 512 tap digital FIR filter capable of providing high levels of filtering functionality.

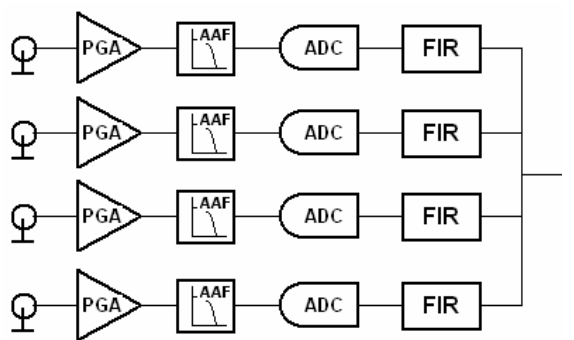

Fig. 3 - Functional circuit blocks for QF4A512.

\subsection{THE MSP430 MICROCONTROLLER}

The QF4A512 is controlled by the MSP430 microcontroller through a Serial Peripheral Interface (SPI) interface. The microcontroller performs control, communication and other intelligent functions. The structure of the software in our experiments is shown in table 1 and is provided by Quickfilter Technologies. The function main.c was modified and two new functions were created.

Table 1. Software structure for msp430

\begin{tabular}{ll|}
\hline Application Code & main.c \\
QF4A512 Functional Driver & Qf4A512-functional.c \\
QF4A512 Access Driver & Qf4A512-access.c \\
Hardware Abstraction & Msp430-SPI.c \\
\hline
\end{tabular}

The QF4A512 SPI interface is en the slave configuration with the microcontroller providing SCLK, see Fig. 1.

\subsection{DIGITAL FILTERING}

The basic function of a filter is to pass frequencies of interest (Pass band) while attenuating everything else (Stop band). Traditional systems perform their filtering function in the analog domain (i. e., prior to digitizing by the ADC). In our approach, filtering is performed after the signal has been converted to ones and zeros using a Digital Signal Processing (DSP), which offers a number of rather compelling advantages. First, the DSP approach completely eliminates noise pickup and also avoids component tolerance and drift issues that plague analog components. Second, DSP solutions are inherently more reproducible and reliable than their analog counterparts. Finally, digital solutions are easy to design, debug and reconfigure.

Figure 4 shows the filter response and impulse response of a FIR filter implemented in our experiments. There are a few factors to consider on filter implementation. See table 2. Most basic is the degree of attenuation between the pass band and stop band. In our example, a filter with $60 \mathrm{~dB}$ of attenuation reduces the amplitude of unwanted frequencies by a factor of 1000 . Another key factor is the selectivity, or quality, of the filter as measured 
by the slope of the roll on and off. The ParksMcClellan filter type offers sharp transition bands at the expense of some pass band ripple, $1 \mathrm{~dB}$. Another factor is the number of taps. Each filter can have up to 512 taps; each tap corresponds to the familiar multiply-and-accumulate (MAC) operation at the core of DSP inner loops.
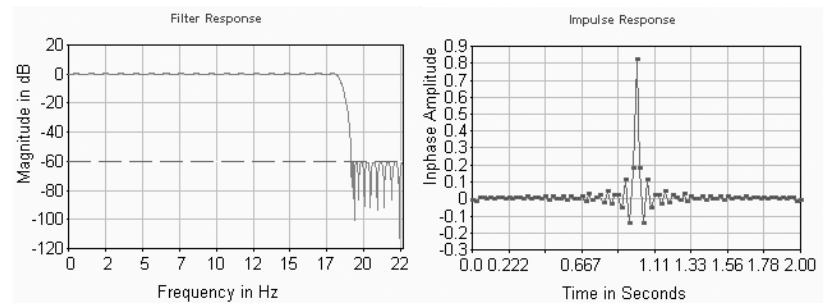

Fig. 4 - Filter response and impulse response.

Table 2. Fir filter

\begin{tabular}{|lc|}
\hline Filter Type & Parks-McClellan \\
Sampling Frequency & $45 \mathrm{~Hz}$ \\
Number of Taps & 91 \\
Coefficient Mode & 20 bit quantized \\
Ripple & $1 \mathrm{~dB}$ \\
Attenuation & $60 \mathrm{~dB}$ \\
Pass Band Upper & $18 \mathrm{~Hz}$ \\
Stop Band Lower & $19 \mathrm{~Hz}$ \\
\hline
\end{tabular}

\section{DIGITAL WIRELESS DATA TRANSMISSION}

For digital wireless data transmission the F2M03GLA Bluetooth module from the company Free2move was used. Bluetooth is a relatively old standard, but it has been updated over the years to increase its efficiency. Bluetooth operates in the unlicensed $2.4 \mathrm{GHz}$ spectrum, with data rates of up to $3 \mathrm{Mbps}$ and has in the past only been able to cover distances of up to 100 metres, but recent progress has increased the range of module up to 30 $\mathrm{km}$.

The F2M03GLA Bluetooth module has a range of $350 \mathrm{~m}$ and uses Bluetooth version 2.0. The main enhancement in this version is the introduction of an Enhanced Data Rate (EDR) of 3.0 Mbits/s that provides the following effects: (1) faster transmission speed; (2) lower power consumption; and (3) simplification of multi-link scenarios due to more available bandwidth. We should note that the F2M03GLA can be replaced by the F2M03GX/GXA Bluetooth module that has a range of up to $1000 \mathrm{~m}$.

The F2M03GLA comes with a standard wireless UART firmware (WU) that implements a Bluetooth Serial Port Profile (SPP). The F2M03GLA in combination with the F2M03G-KIT evaluation kit, see Fig. 1, gives the possibility of easily evaluating the wireless UART firmware as well as customizing it and obtaining access to an extensive range of inputs/outputs ports. The device was properly set up using the Bluetooth configuration software that comes with the product to set connection modes, connect accept settings, security modes, PIN-code, Baud rate, etc.

To test the Bluetooth link, a F2M03G-KIT was connected to the standard computer using the RS232 mode and the other F2M03G-KIT was connected to the MSP430 (on the Digital Data Acquisition System) using the mode TTL. The F2M03G-KIT allows the user to connect to the board using one of the three modes: USB, RS232 or TTL. The Bluetooth link worked properly: the computer was able to communicate with the MSP430 on a TTL level and the commands and data from and to the computer, where data logging and representation were set up, arrived without problems.

\section{RESULTS OF THE EXPERIMENTS}

The system has passed extensive laboratory and field tests (e.g. with man-made explosions). Figure 5 shows the waveform of an infrasound signal generated by opening a door from the four channels.
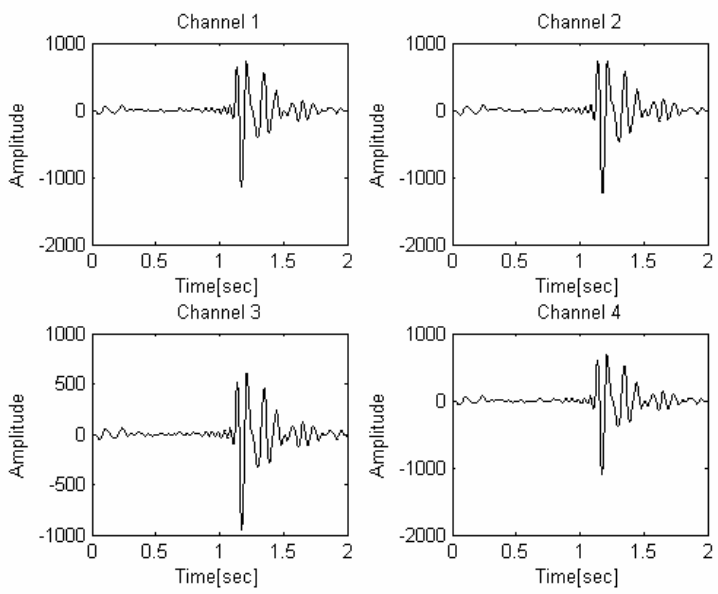

Fig. 5 - Infrasound signal recorded by the four channels.

The four digital signals are summed to get only one signal. Figure 6.a shows the result when summing only channel 1 four times and Fig. 6.b the result when summing the four signals. The corresponding parametric amplitude spectrums are shown by the lower graphs in the same Figure. The Fourier procedure is used to estimate the frequencies and component count. It can be seen that the proposed digital data acquisition system operates satisfactorily in the infrasound band (below $20 \mathrm{~Hz}$ ). 


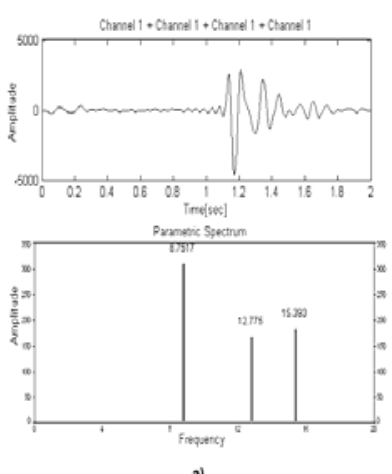

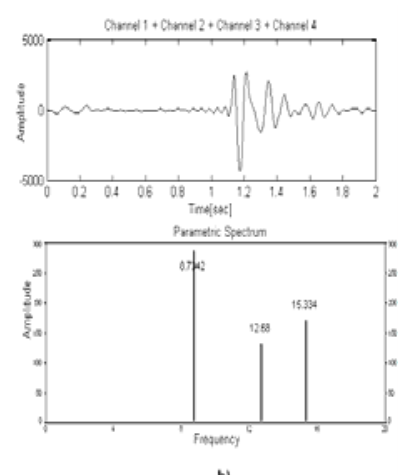

Fig. 6 - The four digital signals are summed to get only one signal.

\section{CONCLUSION}

The design of a digital wireless data acquisition system using a QF4512 programmable signal converter from Quickfilter Technologies, a MSP430 microcontroller from Texas Instruments and a F2M03GLA Bluetooth module from Free2move for infrasonic records is also presented in this thesis. The digital wireless data acquisition system has passed extensive laboratory and field tests.

\section{REFERENCES}

[1] P. Campus, The IMS Infrasound Network and its Potential for Detection of Events: Examples of a Variety of Signals Recorded Around the World, Newsletter No 06, Inframatics, June 2004.

[2] L. Liszka, Cognitive Information Processing in Space Physics and Astrophysics, 2003, Pachart Publishing House, Tucson, Az, USA, ISBN 088126-090-8.

[3] QF4A512 Programmable Signal Converter, Specification and Application Note QFAN004006, 2007 (www.quickfiltertech.com).

[4] C. Phipps, “ Intelligent signal processing at the sensor network edge”, White Paper, July 2005 (www.quickfiltertech.com).

[5] Free2move, 2008, http://www.free2move.se

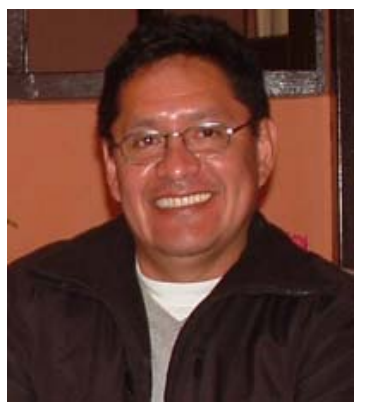

José Chilo was born in Arequipa, Perú in 1960. He received the $M$. Sc. degree in industrial engineering from University of San Agustin Arequipa-Perú in 1986 and the B.S. degree in electrical engineering with specialization in mobile communication systems from Royal Institute of Technology in Sweden. He is a Ph. D. student at Royal Institute of Technology and a lecturer in electronics at University of Gävle in Sweden. His research covers signal processing of infrasound signals.

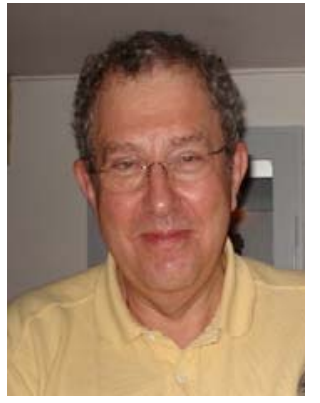

Prof. Thomas Lindblad was born 1945 and got his Ph D in physics from the University of Stockholm in 1974. He became associate professor two years later and continued his research in nuclear spectroscopy, in particular advanced measuring techniques and data analysis.

He has written many papers using neural network hardware in on-line physics experiment as well as using these types of algorithms for off-line analysis or torrent data. For many years he worked with the Manne Siegbahn Institute of Physics, Oak Ridge National Laboratory and ISN, Grenoble. $\mathrm{He}$ is presently professor at the Royal Institute of Technology and Director of Undergraduate Studies in Physics. 